\title{
Bounds on antipodal spherical designs with few angles
}

\author{
Zhiqiang $\mathrm{Xu}^{*} \quad$ Zili $\mathrm{Xu}$ \\ LSEC, Inst. Comp. Math., Academy of Mathematics and System Science \\ Chinese Academy of Sciences \\ Beijing, 100091, China \\ School of Mathematical Sciences \\ University of Chinese Academy of Sciences \\ Beijing, 100049, China \\ $\{$ xuzq,xuzili\}@lsec.cc.ac.cn \\ Wei-Hsuan $\mathrm{Yu}$ \\ Mathematics Department \\ National Central University \\ Taoyuan, Taiwan \\ u690604@gmail.com
}

Submitted: Sep 24, 2020; Accepted: Jun 25, 2021; Published: Aug 13, 2021

(C) The authors. Released under the CC BY-ND license (International 4.0).

\begin{abstract}
A finite subset $X$ on the unit sphere $\mathbb{S}^{d}$ is called an $s$-distance set with strength $t$ if its angle set $A(X):=\{\langle\mathbf{x}, \mathbf{y}\rangle: \mathbf{x}, \mathbf{y} \in X, \mathbf{x} \neq \mathbf{y}\}$ has size $s$, and $X$ is a spherical $t$-design but not a spherical $(t+1)$-design. In this paper, we consider to estimate the maximum size of such antipodal set $X$ for small $s$. Motivated by the method developed by Nozaki and Suda, for each even integer $s \in\left[\frac{t+5}{2}, t+1\right]$ with $t \geqslant 3$, we improve the best known upper bound of Delsarte, Goethals and Seidel. We next focus on two special cases: $s=3, t=3$ and $s=4, t=5$. Estimating the size of $X$ for these two cases is equivalent to estimating the size of real equiangular tight frames (ETFs) and Levenstein-equality packings, respectively. We improve the previous estimate on the size of real ETFs and Levenstein-equality packings. This in turn gives an upper bound on $|X|$ when $s=3, t=3$ and $s=4, t=5$, respectively.
\end{abstract}

Mathematics Subject Classifications: 05E30, 51D20

*Supported by Beijing Natural Science Foundation (Z180002) and by NSFC grant (12025108, $11688101)$. 


\section{Introduction}

\subsection{Spherical designs with few angles}

A finite set $X \subset \mathbb{S}^{d-1}$ is called an $s$-distance set if its angle set $A(X):=\{\langle\mathbf{x}, \mathbf{y}\rangle: \mathbf{x}, \mathbf{y} \in$ $X, \mathbf{x} \neq \mathbf{y}\}$ contains $s$ distinct values, and we say $X$ has strength $t$ if $t$ is the largest integer such that $X$ is a spherical $t$-design. We say that a finite set $X \subset \mathbb{S}^{d-1}$ is a spherical $t$-design if the following equality

$$
\int_{\mathbb{S}^{d-1}} f(\mathbf{x}) d \mu_{d}(\mathbf{x})=\frac{1}{|X|} \sum_{\mathbf{x} \in X} f(\mathbf{x})
$$

holds for any polynomial $f$ of degree at most $t$ (see [12]). Here, $\mu_{d}$ is the Lebesgue measure on $\mathbb{S}^{d-1}$ normalized by $\mu_{d}\left(\mathbb{S}^{d-1}\right)=1$. In this paper we focus on the following problem which originally arises in design theory:

Problem 1. Given $s, t \in \mathbb{Z}_{+}$, what is the maximum size of an $s$-distance set $X \subset \mathbb{S}^{d-1}$ with strength $t$ ?

Spherical designs with few angles usually display beautiful symmetry and optimality $[11,6,16]$, e.g., the universal optimality of the 600 -cell on $\mathbb{S}^{3}[11]$, which have been studied for several decades $[12,3,4]$. Estimating the size of these designs provides a necessary condition on their existence. See $[1,23]$ and the references for the recent work.

In this paper we devote our attention to the antipodal case of this problem, i.e., $X=-X$. We aim to bound the size of antipodal $s$-distance sets in $\mathbb{S}^{d-1}$ with strength $t$. Recall that the strength of an antipodal set must be an odd integer [12, Theorem 5.2]. According to [12, Theorem 6.8], we always have

$$
|X| \leqslant 2\left(\begin{array}{c}
d+s-2 \\
s-1
\end{array}\right) \text { and } 2 s \geqslant t+1
$$

provided $X \subset \mathbb{S}^{d-1}$ is an antipodal $s$-distance set with strength $t$. The upper bound in (1) is called the Delsarte-Goethals-Seidel bound for an antipodal spherical $s$-distance set. Furthermore, the equality in (1) holds if and only if the $s$-distance set $X$ forms a tight spherical $(2 s-1)$-design, i.e., $t+1=2 s$. In this paper we will focus on estimating the size of $X$ when $2 s$ is slightly greater than $t+1$.

\subsection{The optimal line packing problem}

It is particularly interesting to consider two special cases, i.e., $s=3, t=3$ and $s=4, t=$ 5 . These two cases are closely related to the optimal line packing problem, which aims to find a finite set $\Phi=\left\{\boldsymbol{\varphi}_{i}\right\}_{i=1}^{n} \subset \mathbb{S}^{d-1}$ with fixed size $n>d$ and the minimal coherence $\mu(\Phi):=\max _{i \neq j}\left|\left\langle\boldsymbol{\varphi}_{i}, \boldsymbol{\varphi}_{j}\right\rangle\right|$ (see $\left.[10,13,15,17]\right)$. The followings are two well-known lower 
bounds on the coherence:

$$
\begin{aligned}
& \mu(\Phi) \geqslant \sqrt{\frac{n-d}{d(n-1)}}, \quad \text { if } n>d, \\
& \mu(\Phi) \geqslant \sqrt{\frac{3 n-d(d+2)}{(d+2)(n-d)}}, \text { if } n>\frac{d(d+1)}{2} .
\end{aligned}
$$

The (2a) is called the Welch bound [31] and the $(2 \mathrm{~b})$ is called the Levenstein bound $[19,20]$. It is well known that the equality in (2a) occurs when $\Phi \cup-\Phi$ forms an antipodal 3 -distance 3 -strength set or an antipodal 3-distance 5-strength set with size $d(d+1)[12$, Example 8.3]; and the equality in (2b) occurs when $\Phi \cup-\Phi$ forms an antipodal 4-distance 5 -strength set or an antipodal 4-distance 7-strength set with size $\frac{d(d+1)(d+2)}{3}[12$, Example 8.4]. Hence, estimating the size of the antipodal 3-distance 3-strength sets and of the antipodal 4-distance 5-strength sets is helpful to know the existence of these two kinds of optimal packings. In the context of frame theory, a set achieving the Welch bound in (2a) is known as a real equiangular tight frame (ETF). Hence, bounding the size of an antipodal 3-distance set with strength 3 is equivalent to bounding the size of a real ETF whose size is strictly smaller than $\frac{d(d+1)}{2}$. This is particularly interesting since the existence of real ETFs is a long-standing open problem for most pairs $(d, n)[14,25]$. For the nontrivial case where $n>d+1>2$, an ETF may exist only if its size $n$ satisfies the Gerzon bound $[18,14,13]$ :

$$
d+\frac{1}{2}+\sqrt{2 d+\frac{1}{4}} \leqslant n \leqslant \frac{d(d+1)}{2} .
$$

\subsection{Related work}

We overview the known upper bounds on antipodal spherical designs with few angles. Let $X \subset \mathbb{S}^{d-1}$ be an antipodal $s$-distance set with strength $t$. As said before, $t$ must be an odd integer [12, Theorem 5.2]. Set

$$
h_{0}:=1, h_{1}:=d, h_{k}:=\left(\begin{array}{c}
d+k-1 \\
k
\end{array}\right)-\left(\begin{array}{c}
d+k-3 \\
k-2
\end{array}\right), k \geqslant 2
$$

and

$$
\delta_{s}:= \begin{cases}0, & \text { if } s \text { is even, } \\ 1, & \text { if } s \text { is odd }\end{cases}
$$

For each odd integer $t \in\left[s-\delta_{s}-1,2 s-2 \delta_{s}-3\right]$, Nozaki and Suda in [23, Corollary 3.7] derived a new upper bound on $|X|$ :

$$
|X| \leqslant 2\left(\begin{array}{c}
d+s-\delta_{s}-1 \\
s-\delta_{s}
\end{array}\right)-2 h_{t-s+\delta_{s}+1}
$$

If $s \geqslant 3$ is odd and $t \in[s-2,2 s-5]$, it is easy to see that the bound in (6) lowers the Delsarte-Goethals-Seidel bound by $2 h_{t-s+2}$. If $s \geqslant 2$ is even and $t \in[s-1,2 s-3]$, the 
upper bound in (6) becomes $2\left(\begin{array}{c}d+s-1 \\ s\end{array}\right)-2 h_{t-s+1}$. When $s$ is fixed, a simple calculation shows that $2\left(\begin{array}{c}d+s-1 \\ s\end{array}\right)-2 h_{t-s+1}=\Theta\left(d^{s}\right)$ while the Delsarte-Goethals-Seidel bound in (1) is $\Theta\left(d^{s-1}\right)$. Hence, if $s$ is an fixed even integer and $d$ is large enough, the upper bound in (6) is larger than the Delsarte-Goethals-Seidel bound.

\subsection{Our contributions}

Assume that $X \subset \mathbb{S}^{d-1}$ is an antipodal $s$-distance set with strength $t$. The aim of this paper is to present a better upper bound on $|X|$.

\subsubsection{The general case}

Motivated by the methods developed in [23], we present an upper bound for $|X|$ which lowers the Delsarte-Goethals-Seidel bound when $s \in\left[\frac{t+5}{2}, t+1\right]$ is an even integer and $t \geqslant 3$.

Theorem 2. Let $d \geqslant 2$ be an integer. Assume that $X \subset \mathbb{S}^{d-1}$ is an antipodal s-distance set with strength $t \geqslant 3$, where $s \in\left[\frac{t+5}{2}, t+1\right]$ is an even integer. Then, we have

$$
|X| \leqslant 2\left(\begin{array}{c}
d+s-2 \\
s-1
\end{array}\right)-2 h_{t-s+2}
$$

where $h_{k}$ is defined in (4) for each $k \geqslant 0$.

We next consider to estimate $|X|$ for the case when $s=\frac{t+3}{2}$. We mainly focus on two special cases : $s=3, t=3$ and $s=4, t=5$.

\subsubsection{The case: $s=3, t=3$}

As mentioned before, a set $\Phi$ is an ETF for $\mathbb{R}^{d}$ with size $n<\frac{d(d+1)}{2}$ if and only if $\Phi \cup-\Phi$ is an antipodal 3-distance sets with strength 3 [12, Example 8.3]. Hence, we direct our attention to estimating the size of real ETFs. The following theorem presents a necessary condition for the size of real ETFs.

Theorem 3. Let $d \geqslant 5$ be an integer. Assume that $\Phi$ is an ETF for $\mathbb{R}^{d}$ with size $n>d+1$. Then, we have either $n \in\left\{d+\frac{1}{2}+\sqrt{2 d+\frac{1}{4}}, \frac{d(d+1)}{2}\right\}$ or

$$
d+\frac{1}{2}+\sqrt{3 d+\frac{1}{4}} \leqslant n \leqslant \frac{d(d+2)}{3} .
$$

Theorem 3 improves the Gerzon bound (3) when $n \notin\left\{d+\frac{1}{2}+\sqrt{2 d+\frac{1}{4}}, \frac{d(d+1)}{2}\right\}$. To our knowledge, one only finds two pairs $(d, n)$ for which ETFs exist and achieve the size $\frac{d(d+2)}{3}:(6,16)$ and $(22,176)$. The known configuration of these two ETFs is a subset of ETFs with parameters $(7,28)$ and $(23,276)$, respectively (see [28, Remark 5.2] and [27, Page 271]). Also, one only finds two pairs $(d, n)$ for which ETFs exist and achieve 
the size $d+\frac{1}{2}+\sqrt{3 d+\frac{1}{4}}:(10,16)$ and $(154,176)$. These two ETFs are the Naimark complements of ETFs with parameters $(6,16)$ and $(22,176)$, respectively [14]. Motivated by the observation, we present the following conjecture:

Conjecture 4. Assume that $d \geqslant 5$. There exists an ETF with parameters $\left(d+1, \frac{(d+1)(d+2)}{2}\right)$ if and only if there exists an ETF with parameters $\left(d, \frac{d(d+2)}{3}\right)$.

Recall that $X \subset \mathbb{S}^{d-1}$ is an antipodal 3-distance set with strength 3 if and only if $X=\Phi \cup-\Phi$ where $\Phi$ is an ETF in $\mathbb{R}^{d}$ with size $n<\frac{d(d+1)}{2}$. A simple observation is $\Phi \cap-\Phi=\emptyset$ if $\Phi$ is an ETF. Hence, we immediately obtain an upper bound for the antipodal 3-distance sets with strength 3 .

Corollary 5. Let $d \geqslant 5$ be an integer. Assume $X \subset \mathbb{S}^{d-1}$ is an antipodal 3-distance set with strength 3 . Then, we have either $|X| \in\{2 d+2,2 d+1+\sqrt{8 d+1}\}$ or

$$
2 d+1+\sqrt{12 d+1} \leqslant|X| \leqslant \frac{2 d(d+2)}{3} .
$$

Remark 6. According to Delsarte-Goethals-Seidel bound in (1), $|X| \leqslant d(d+1)$ if $X \subset \mathbb{S}^{d-1}$ is an antipodal 3-distance set with strength 3 . Corollary 5 lowers the bound to $\frac{2 d(d+2)}{3}$.

We next introduce another result on the existence of real ETFs. It is well known that the existence of a real ETF for $\mathbb{R}^{d}$ with size $n>d+1>2$ is equivalent to the existence of a strongly regular graph with parameters $\left(n-1, a, \frac{3 a-n}{2}, \frac{a}{2}\right)[25,29,14]$, where

$$
a:=\frac{n}{2}-1+\left(1-\frac{n}{2 d}\right) \sqrt{\frac{d(n-1)}{n-d}} .
$$

Since every strongly regular graph satisfies the Krein conditions (see Lemma 21 for details), one is interested in whether the Krein conditions are covered by the Gerzon bound (3) or other known necessary conditions (see [29, 14]). In Proposition 22 we will give a positive answer to this question showing that the Krein conditions for strongly regular graphs with parameters $\left(n-1, a, \frac{3 a-n}{2}, \frac{a}{2}\right)$ are equivalent to the Gerzon bound (3).

\subsubsection{The case: $s=4, t=5$}

Finally, we consider to estimate the size of antipodal 4-distance sets with strength 5 . Recall that the Levenstein bound $(2 \mathrm{~b})$ is attained only if $n>\frac{d(d+1)}{2}$ [20, Theorem 6.13]. Moreover, $\Phi \subset \mathbb{S}^{d-1}$ is a Levenstein-equality packing with size $n<\frac{d(d+1)(d+2)}{6}$ if and only if $\Phi \cup-\Phi$ is an antipodal 4-distance set with strength 5 [12, Example 8.4]. Thus, we mainly focus on estimating the size of Levenstein-equality packings.

We begin with providing an estimate on the size of Levenstein-equality packings, i.e., $\mu(\Phi)=\sqrt{\frac{3 n-d(d+2)}{(d+2)(n-d)}}$. 
Theorem 7. Let $d \geqslant 4$ be an integer. Assume $\Phi \subset \mathbb{S}^{d-1}$ is a Levenstein-equality packing with size $n$. Then we have either $n=\frac{d(d+2)}{2}$ or

$$
n \in\left\{\frac{d(d+2)(d-1+\alpha)}{3 \alpha}: \alpha \in\left[2, \frac{2(d-1)(d+2)}{d+5}\right] \cap \mathbb{Z}\right\} \cap \mathbb{Z}
$$

Particularly, if $\Phi \subset \mathbb{S}^{d-1}$ is a Levenstein-equality packing with size $n \notin\left\{\frac{d(d+2)}{2}, \frac{d(d+1)(d+2)}{6}\right\}$ then we have $n \in\left[\frac{d(d+3)}{2}, \frac{d(d+2)^{2}}{9}\right]$.

Remark 8. Taking $\alpha=\frac{2(d-1)(d+2)}{d+5}$ in (10), we have $n=\frac{d(d+3)}{2}$. To our knowledge, there does not exist a Levenstein-equality packing in $\mathbb{R}^{d}$ with size $\frac{d(d+3)}{2}$. Taking $\alpha=3$ in (10), we have $n=\frac{d(d+2)^{2}}{9}$. To our knowledge, so far, one only finds two pairs $(d, n)$ for which Levenstein-equality packings achieve the size $\frac{d(d+2)^{2}}{9}:(7,63)$ and $(22,1408)$. The corresponding packings come from a tight spherical 7-design in $\mathbb{R}^{8}$ and $\mathbb{R}^{23}$, respectively [21, Page 619]. We next explain the link between tight spherical 7-designs and Levensteinequality packings. Assume that $X \subset \mathbb{R}^{d+1}$ is a tight spherical 7-design. According to [12, Theorem 8.2] there exists a $\mathbf{x} \in X$ so that the derived code $Z:=\{\mathbf{y} \in X:\langle\mathbf{y}, \mathbf{x}\rangle=0\}$ forms an antipodal 4 -distance 5 -strength set in $\mathbb{R}^{d}$ with the angle set $\left\{-1,0, \pm \sqrt{\frac{3}{d+5}}\right\}$. Hence, choosing a point from each antipodal pair of points in $Z$ gives a Levenstein-equality packing $\Phi \subset \mathbb{S}^{d-1}$. Combining $\mu(\Phi)=\sqrt{\frac{3}{d+5}}$ with $(2 \mathrm{~b})$ we obtain that $\Phi$ has size $\frac{d(d+2)^{2}}{9}$. Hence, if we have a tight spherical 7-design in $\mathbb{R}^{d+1}$, we can obtain a Levenstein-equality packings in $\mathbb{R}^{d}$ with size $\frac{d(d+2)^{2}}{9}$. We are interested in knowing whether each Levensteinequality packing with size $\frac{d(d+2)^{2}}{9}$ comes from a tight spherical 7-design.

Remind that $X \subset \mathbb{S}^{d-1}$ is an antipodal 4-distance set with strength 5 if and only if $X=\Phi \cup-\Phi$ where $\Phi$ is a Levenstein-equality packing in $\mathbb{R}^{d}$ with size $n<\frac{d(d+1)(d+2)}{6}$. Based on Theorem 7, we have the following corollary:

Corollary 9. Let $d \geqslant 4$ be an integer. Assume $X \subset \mathbb{S}^{d-1}$ is an antipodal 4-distance set with strength 5. Then, we have either $|X|=d(d+2)$ or

$$
|X| \in\left\{\frac{2 d(d+2)(d-1+\alpha)}{3 \alpha}: \alpha \in\left[3, \frac{2(d-1)(d+2)}{d+5}\right] \cap \mathbb{Z}\right\} \cap \mathbb{Z} .
$$

Remark 10. According to (11), we obtain that $|X| \leqslant \frac{2 d(d+2)^{2}}{9}$ if $X \subset \mathbb{S}^{d-1}$ is an antipodal 4-distance set with strength 5. Recall that the Delsarte-Goethals-Seidel bound in (1) gives $|X| \leqslant \frac{d(d+1)(d+2)}{3}$ when $s=4$, and that the Nozaki-Suda bound in (6) gives $|X| \leqslant$ $\frac{1}{12} \cdot(d+2)\left(d^{3}+4 d^{2}-9 d+12\right)$ when $s=4, t=5$. A simple calculation shows that

$$
\frac{2 d(d+2)^{2}}{9}<\frac{d(d+1)(d+2)}{3}<\frac{(d+2)\left(d^{3}+4 d^{2}-9 d+12\right)}{12}, \quad \text { for } d \geqslant 4 .
$$

Hence, the upper bound $|X| \leqslant \frac{2 d(d+2)^{2}}{9}$ improves both the Nozaki-Suda bound and the Delsarte-Goethals-Seidel bound when $d \geqslant 4$. 
In Table 1 we summarize the best known upper bounds on the antipodal $s$-distance $t$-strength sets so far.

Table 1: Upper bounds on the size of an antipodal $s$-distance set $X \subset \mathbb{S}^{d-1}$ with strength $t$

\begin{tabular}{c|c} 
The values of $s$ and $t$ & An upper bound of $|X|$ \\
\hline$s=3, t=3$ & $\frac{2 d(d+2)}{3}($ Corollary 5$)$ \\
\hline$s=4, t=5$ & $\frac{2 d(d+2)^{2}}{9}($ Corollary 9$)$ \\
\hline even $s=\frac{t+3}{2}, t \geqslant 7$ & $\min \left\{2\left(\begin{array}{c}d+s-2 \\
s-1\end{array}\right), 2\left(\begin{array}{c}d+s-1 \\
s\end{array}\right)-2 h_{t-s+1}\right\}([12],[23])$ \\
\hline even $s \in\left[\frac{t+5}{2}, t+1\right], t \geqslant 3$ & $2\left(\begin{array}{c}d+s-2 \\
s-1\end{array}\right)-2 h_{t-s+2}$ (Theorem 2) \\
\hline odd $s \in\left[\frac{t+5}{2}, t+2\right]$ & $2\left(\begin{array}{c}d+s-2 \\
s-1\end{array}\right)-2 h_{t-s+2}[23]$
\end{tabular}

\subsection{Organization}

The paper is organized as follows. In Section 2, we introduce some definitions and lemmas. After presenting the proof of Theorem 2 in Section 3, we prove Theorem 3 in Section 4. We also show the equivalence between the Gerzon bound and the necessary conditions on the existence of real ETFs obtained from the Krein conditions in Section 4. Finally we prove Theorem 7 in Section 5.

\section{Preliminaries}

In this section, we introduce some definitions and lemmas which will be used in later sections.

\section{$2.1 \quad$ Notations}

Let $\operatorname{Harm}_{k}\left(\mathbb{R}^{d}\right)$ be the vector space of all real homogeneous harmonic polynomials of degree $k$ on $d$ variables, equipped with the standard inner product

$$
\langle f, g\rangle=\int_{\mathbb{S}^{d-1}} f(\mathbf{x}) g(\mathbf{x}) \mathrm{d} \mu_{d}(\mathbf{x})
$$

for $f, g \in \operatorname{Harm}_{k}\left(\mathbb{R}^{d}\right)$. It is known that the dimension of $\operatorname{Harm}_{k}\left(\mathbb{R}^{d}\right)$ is $h_{k}$, where $h_{k}$ is defined in (4) for each $k \geqslant 0\left[12\right.$, Theorem 3.2]. Let $\left\{\phi_{k, i}^{(d)}\right\}_{i=1}^{h_{k}}$ be an orthonormal basis for $\operatorname{Harm}_{k}\left(\mathbb{R}^{d}\right)$.

Let $G_{k}^{(d)}(x)$ denote the Gegenbauer polynomial of degree $k$ with the normalization $G_{k}^{(d)}(1)=h_{k}$, which can be defined recursively as follows (see also [12, Definition 2.1]):

$$
\begin{gathered}
G_{0}^{(d)}(x):=1, G_{1}^{(d)}(x):=d \cdot x \\
\frac{k+1}{d+2 k} \cdot G_{k+1}^{(d)}(x)=x \cdot G_{k}^{(d)}(x)-\frac{d+k-3}{d+2 k-4} \cdot G_{k-1}^{(d)}(x), \quad k \geqslant 1 .
\end{gathered}
$$


The following formulation is well-known [12, Theorem 3.3]:

$$
G_{k}^{(d)}(\langle\mathbf{x}, \mathbf{y}\rangle)=\sum_{i=1}^{h_{k}} \phi_{k, i}^{(d)}(\mathbf{x}) \phi_{k, i}^{(d)}(\mathbf{y}), \quad \text { for } \quad \mathbf{x}, \mathbf{y} \in \mathbb{S}^{d-1}, k \in \mathbb{Z}_{+} .
$$

We also need the following notations.

Definition 11. For a finite non-empty set $X \subset \mathbb{S}^{d-1}$, we use the following notations:

1. The $k$-th characteristic matrix $\mathbf{H}_{k}(X)$ of size $|X| \times h_{k}$ is defined as (see also [12, Definition 3.4]):

$$
\mathbf{H}_{k}(X):=\left(\phi_{k, i}^{(d)}(\mathbf{x})\right), \mathbf{x} \in X, i \in\left\{1,2, \ldots, h_{k}\right\}
$$

2. Set $\mathbf{D}_{k}(X):=\mathbf{H}_{k}(X) \mathbf{H}_{k}(X)^{\mathrm{T}}$ for each $k \geqslant 0$;

3. Let $V_{k}(X)$ denote the direct sum of the eigenspaces corresponding to all positive eigenvalues of $\mathbf{D}_{k}(X)$ (see also [23, Page 1707]);

4. The annihilator polynomial of $X$ is defined as

$$
F_{X}(x):=\prod_{\alpha \in A(X)} \frac{x-\alpha}{1-\alpha}
$$

5. When $X$ is antipodal, we say the subset $\hat{X} \subset X$ is a half of $X$ if $\hat{X}$ satisfies $\hat{X} \cap-\hat{X}=\varnothing$ and $\hat{X} \cup-\hat{X}=X$.

Note that $\mathbf{H}_{0}(X)$ is exactly the all-ones vector of size $|X|$. According to (12), we have

$$
\mathbf{D}_{k}(X)=\mathbf{H}_{k}(X) \mathbf{H}_{k}(X)^{\mathrm{T}}=\left(G_{k}^{(d)}(\langle\mathbf{x}, \mathbf{y}\rangle)\right)_{\mathbf{x}, \mathbf{y} \in X} .
$$

Throughout this paper, we use $\mathbf{I}, \mathbf{J}$ to denote the identity matrix and all-ones matrix of appropriate size, respectively. We also set

$$
\boldsymbol{\Delta}_{k, l}:= \begin{cases}\mathbf{I}, & \text { if } k=l \\ \mathbf{0}, & \text { otherwise. }\end{cases}
$$

\subsection{Spherical designs}

By the notion of characteristic matrices, the following lemma provides two equivalent definitions of spherical $t$-designs.

Lemma 12. (see [12, Theorem 5.3]) A finite set $X \subset \mathbb{S}^{d-1}$ is a spherical t-design if and only if any one of the following holds:

1. $\mathbf{H}_{k}(X)^{\mathrm{T}} \mathbf{H}_{0}(X)=\mathbf{0}_{h_{k} \times 1}, k=1,2, \ldots, t$. 
2. $\mathbf{H}_{k}(X)^{\mathrm{T}} \mathbf{H}_{l}(X)=|X| \cdot \boldsymbol{\Delta}_{k, l}$ when $0 \leqslant k+l \leqslant t$.

We next prove some properties of antipodal spherical designs which will be used in Section 3.

Corollary 13. Assume $X \subset \mathbb{S}^{d-1}$ is an antipodal set and let $\hat{X}$ be a half of $X$. Then,

1. $X$ is a spherical $t$-design if and only if $\mathbf{H}_{k}(\hat{X})^{\mathrm{T}} \mathbf{H}_{0}(\hat{X})=\mathbf{0}_{h_{k} \times 1}$ for each positive even integer $k \leqslant t$.

2. if $X$ is a spherical t-design, then

$$
\mathbf{H}_{k}(\hat{X})^{\mathrm{T}} \mathbf{H}_{l}(\hat{X})=\frac{|X|}{2} \cdot \boldsymbol{\Delta}_{k, l} \quad \text { and } \quad \mathbf{D}_{k}(\hat{X}) \mathbf{D}_{l}(\hat{X})=\frac{|X|}{2} \cdot \boldsymbol{\Delta}_{k, l} \cdot \mathbf{D}_{k}(\hat{X})
$$

hold when $0 \leqslant k+l \leqslant t$ and $k \equiv l(\bmod 2)$.

Proof. For any $\mathbf{x} \in \mathbb{S}^{d-1}$ and $i \in\left\{1,2, \ldots, h_{k}\right\}$, we have $\phi_{k, i}^{(d)}(-\mathbf{x})=-\phi_{k, i}^{(d)}(\mathbf{x})$ if $k$ is odd and $\phi_{k, i}^{(d)}(-\mathbf{x})=\phi_{k, i}^{(d)}(\mathbf{x})$ if $k$ is even. Hence, we have

$$
\mathbf{H}_{k}(X)=\left(\begin{array}{c}
\mathbf{H}_{k}(\hat{X}) \\
(-1)^{k} \cdot \mathbf{H}_{k}(\hat{X})
\end{array}\right)
$$

(i) According to (14) we have

$$
\mathbf{H}_{k}(X)^{\mathrm{T}} \mathbf{H}_{0}(X)= \begin{cases}2 \cdot \mathbf{H}_{k}(\hat{X})^{\mathrm{T}} \mathbf{H}_{0}(\hat{X}), & \text { if } k \text { is even } \\ \mathbf{0}_{h_{k} \times 1}, & \text { if } k \text { is odd }\end{cases}
$$

Based on Lemma 12 we obtain that $X$ is a spherical $t$-design if and only if $\mathbf{H}_{k}(\hat{X})^{\mathrm{T}} \mathbf{H}_{0}(\hat{X})=$ $\mathbf{0}_{h_{k} \times 1}$ for each positive even integer $k \leqslant t$.

(ii) Let $k$ and $l$ be two integers satisfying $0 \leqslant k+l \leqslant t$ and $k \equiv l(\bmod 2)$. Equation (14) implies $\mathbf{H}_{k}(X)^{\mathrm{T}} \mathbf{H}_{l}(X)=2 \cdot \mathbf{H}_{k}(\hat{X})^{\mathrm{T}} \mathbf{H}_{l}(\hat{X})$. Thus, according to Lemma 12 we obtain that $\mathbf{H}_{k}(\hat{X})^{\mathrm{T}} \mathbf{H}_{l}(\hat{X})=\frac{|X|}{2} \cdot \boldsymbol{\Delta}_{k, l}$ if $X$ is a spherical $t$-design. According to (2) in Definition 11 , we have

$$
\mathbf{D}_{k}(\hat{X}) \mathbf{D}_{l}(\hat{X})=\mathbf{H}_{k}(\hat{X}) \mathbf{H}_{k}(\hat{X})^{T} \mathbf{H}_{l}(\hat{X}) \mathbf{H}_{l}(\hat{X})^{T}=\frac{|X|}{2} \cdot \boldsymbol{\Delta}_{k, l} \cdot \mathbf{D}_{k}(\hat{X}) .
$$

The following lemma played a key role in Nozaki and Suda's framework [23]. Its main idea is to identify the size of an $s$-distance set $X$ with the dimension of a sum of subspaces $V_{k}(X)$ defined in Definition 11.

Lemma 14. (see [23, Lemma 3.2]) Let $X \subset \mathbb{S}^{d-1}$ be an s-distance set. Assume the annihilator polynomial of $X$ has the Gegenbauer expansion $F_{X}(x)=\sum_{k=0}^{s} f_{k} G_{k}^{(d)}(x)$. Then we have $|X|=\operatorname{dim}\left(\sum_{k: f_{k}>0} V_{k}(X)\right)$. 


\subsection{Spherical embeddings of strongly regular graphs}

In this subsection we briefly introduce the spherical embeddings of strongly regular graphs, which will be used in our analysis of Levenstein-equality packings in Section 5. A regular graph $\Gamma$ with $v$ vertices and degree $k$ is called strongly regular if every two adjacent vertices have $\lambda$ common neighbors and every two non-adjacent vertices have $\mu$ common neighbors. Let $\Gamma$ be a strongly regular graph with parameters $(v, k, \lambda, \mu)$. Denote its vertex set by $\{1,2, \ldots, v\}$ for simplicity. The adjacency matrix $\mathbf{A}$ of $\Gamma$ has three eigenvalues $k, r_{1}$ and $r_{2}$, with multiplicities $1, n_{1}$ and $n_{2}$, respectively. The values of $r_{1}, r_{2}, n_{1}, n_{2}$ can be calculated as follows $[7,9]$

$$
\begin{aligned}
& r_{1}=\frac{1}{2}\left(\lambda-\mu+\sqrt{(\lambda-\mu)^{2}+4(k-\mu)}\right), \quad r_{2}=\frac{1}{2}\left(\lambda-\mu-\sqrt{(\lambda-\mu)^{2}+4(k-\mu)}\right), \\
& n_{1}=\frac{1}{2}\left(v-1-\frac{2 k+(v-1)(\lambda-\mu)}{\sqrt{(\lambda-\mu)^{2}+4(k-\mu)}}\right), \quad n_{2}=\frac{1}{2}\left(v-1+\frac{2 k+(v-1)(\lambda-\mu)}{\sqrt{(\lambda-\mu)^{2}+4(k-\mu)}}\right) .
\end{aligned}
$$

For each $i \in\{1,2\}$, let $E_{i}$ denote the eigenspace of $\mathbf{A}$ with respect to the eigenvalue $r_{i}$. Then a spherical embedding of $\Gamma$ with respect to $E_{i}$ is a collection of unit vectors in $\mathbb{R}^{n_{i}}$, obtained by orthogonally projecting a standard basis of $\mathbb{R}^{v}$ onto the eigenspace $E_{i}$ and rescaling the projections to have unit norm. It is known that the obtained set is a two-distance spherical 2-design [7,9]. If we let $Y^{(i)}=\left\{\mathbf{y}_{j}^{(i)}\right\}_{j=1}^{v}$ denote the spherical embedding of $\Gamma$ with respect to $E_{i}, i \in\{1,2\}$, then we have $[7,9,5]$ :

$$
\left|\left\langle\mathbf{y}_{j}^{(i)}, \mathbf{y}_{l}^{(i)}\right\rangle\right|=\left\{\begin{array}{cl}
1, & \text { if } j=l, \\
\frac{r_{i}}{k}, & \text { if vertex } j \text { and vertex } l \text { are adjacency, } \\
-\frac{r_{i}+1}{v-k-1}, & \text { otherwise. }
\end{array}\right.
$$

In Section 5 we will introduce that each Levenstein-equality packing gives rise to a strongly regular graph. Then we will use one of the spherical embeddings of this strongly regular graph to provide a lower bound on the size of Levenstein-equality packings.

\section{Proof of Theorem 2}

In this section, motivated by the method developed in [23], we present a proof of Theorem 2.

Assume $s$ is an even integer. Let $X \subset \mathbb{S}^{d-1}$ be an antipodal $s$-distance set with strength $t$ and let $\hat{X}$ be a half of $X$ (see (5) in Definition 11). Now we focus on estimating the maximum size of $\hat{X}$. Noting that $X$ is antipodal and $s$ is even, we assume $A(X)=$ $\left\{-1,0, \pm \alpha_{1}, \ldots, \pm \alpha_{\frac{s-2}{2}}\right\}$, where $\alpha_{i} \in(0,1)$ for each $i \in\left\{1,2, \ldots, \frac{s-2}{2}\right\}$. Noting that $A(\hat{X})=A(X) \backslash\{-1\}$, we have

$$
F_{\hat{X}}(x)=x \cdot \prod_{i=1}^{\frac{s-2}{2}} \frac{x^{2}-\alpha_{i}^{2}}{1-\alpha_{i}^{2}}
$$


It follows that $F_{\hat{X}}(x)$ is an odd function. Assume that $F_{\hat{X}}(x)$ has the Gegenbauer expansion $F_{\hat{X}}(x)=\sum_{k=0}^{s-1} f_{k} G_{k}^{(d)}(x)$. It is well known that the Gegenbauer polynomial $G_{k}^{(d)}(x)$ is an odd function if $k$ is odd and an even function if $k$ is even [26, Page 59]. This means that $f_{k}=0$ provided $k \leqslant s-1$ is even. Hence, by Lemma 14 we obtain

$$
|\hat{X}|=\operatorname{dim}\left(\sum_{k: f_{k}>0} V_{k}(\hat{X})\right) \leqslant \operatorname{dim}\left(\sum_{k=0}^{\frac{s-2}{2}} V_{2 k+1}(\hat{X})\right) .
$$

Now we aim to prove that $V_{t-s+2}(\hat{X})$ is contained in the sum of some other subspaces $V_{2 k+1}(\hat{X})$ when $s \in\left[\frac{t+5}{2}, t+1\right]$. The following lemma is analogous to [23, Lemma 3.3].

Lemma 15. Suppose $X \subset \mathbb{S}^{d-1}$ is an antipodal s-distance set with strength $t$, where $s \in\left[\frac{t+5}{2}, t+1\right]$ is an even integer and $t \geqslant 3$ is an odd integer. Let $\hat{X}$ be a half of $X$. Assume the annihilator polynomial of $\hat{X}$ has the Gegenbauer expansion $F_{\hat{X}}(x)=\sum_{k=0}^{\frac{s-2}{2}} f_{2 k+1} G_{2 k+1}^{(d)}(x)$. If $f_{2 i+1} \neq \frac{1}{|\hat{X}|}$ for some integer $i$ satisfying $t-s+2 \leqslant 2 i+1 \leqslant \frac{t-1}{2}$, then we have $V_{2 i+1}(\hat{X}) \subset \sum_{k=\frac{t-2 i-1}{2}}^{\frac{s-2}{2}} V_{2 k+1}(\hat{X})$.

Proof. Set $\mathbf{F}:=\left(F_{\hat{X}}(\langle\mathbf{x}, \mathbf{y}\rangle)\right)_{\mathbf{x}, \mathbf{y} \in \hat{X}}$. Noting that $F_{\hat{X}}(1)=1$ and $F_{\hat{X}}(\alpha)=0$ for $\alpha \in A(\hat{X})$, we obtain that $\mathbf{F}$ is exactly the identity matrix of size $|\hat{X}|$. On the other hand, by the Gegenbauer expansion of $F_{\hat{X}}(x)$ and (13), we have

$$
\mathbf{I}=\mathbf{F}=\sum_{k=0}^{\frac{s-2}{2}} f_{2 k+1} \cdot \mathbf{D}_{2 k+1}(\hat{X})
$$

For each integer $i$ satisfying $t-s+2 \leqslant 2 i+1 \leqslant \frac{t-1}{2}$, we multiply $\mathbf{D}_{2 i+1}(\hat{X})$ on both sides of (19) and obtain

$$
\begin{aligned}
\mathbf{D}_{2 i+1}(\hat{X}) & =\sum_{k=0}^{\frac{s-2}{2}} f_{2 k+1} \cdot \mathbf{D}_{2 k+1}(\hat{X}) \mathbf{D}_{2 i+1}(\hat{X}) \\
& =f_{2 i+1} \cdot \mathbf{D}_{2 i+1}(\hat{X}) \mathbf{D}_{2 i+1}(\hat{X})+\sum_{k=0, k \neq i}^{\frac{s-2}{2}} f_{2 k+1} \cdot \mathbf{D}_{2 k+1}(\hat{X}) \mathbf{D}_{2 i+1}(\hat{X}) .
\end{aligned}
$$

Since $X$ is an antipodal spherical $t$-design, by Corollary 13, we have

$$
\mathbf{D}_{2 i+1}(\hat{X})=|\hat{X}| \cdot f_{2 i+1} \cdot \mathbf{D}_{2 i+1}(\hat{X})+\sum_{k=\frac{t-2 i-1}{2}}^{\frac{s-2}{2}} f_{2 k+1} \cdot \mathbf{D}_{2 k+1}(\hat{X}) \mathbf{D}_{2 i+1}(\hat{X}) .
$$


Noting that $s \in\left[\frac{t+5}{2}, t+1\right]$ is an even integer and $t \geqslant 3$ is an odd integer, rearranging equation (21) gives

$$
\left(1-|\hat{X}| \cdot f_{2 i+1}\right) \mathbf{D}_{2 i+1}(\hat{X})=\sum_{k=\frac{t-2 i-1}{2}}^{\frac{s-2}{2}} f_{2 k+1} \cdot \mathbf{D}_{2 k+1}(\hat{X}) \mathbf{D}_{2 i+1}(\hat{X}) .
$$

Assume $\mathbf{v}$ is an eigenvector of $\mathbf{D}_{2 i+1}(\hat{X})$ with respect to an eigenvalue $\lambda \neq 0$. Then, we have

$$
\left(1-|\hat{X}| \cdot f_{2 i+1}\right) \mathbf{D}_{2 i+1}(\hat{X}) \mathbf{v}=\sum_{k=\frac{t-2 i-1}{2}}^{\frac{s-2}{2}} f_{2 k+1} \cdot \mathbf{D}_{2 k+1}(\hat{X}) \mathbf{D}_{2 i+1}(\hat{X}) \mathbf{v}
$$

which implies

$$
\left(1-|\hat{X}| \cdot f_{2 i+1}\right) \cdot \lambda \mathbf{v}=\lambda \cdot \sum_{k=\frac{t-2 i-1}{2}}^{\frac{s-2}{2}} f_{2 k+1} \cdot \mathbf{D}_{2 k+1}(\hat{X}) \mathbf{v}
$$

Note that a real symmetric matrix of size $|\hat{X}|$ always has $|\hat{X}|$ linear independent eigenvectors. Hence, we can write $\mathbf{v}=\sum_{j=1}^{|\hat{X}|} \mathbf{v}_{j}^{(2 k+1)}$ for each $k \in\left[\frac{t-2 i-1}{2}, \frac{s-2}{2}\right]$, where $\left\{\mathbf{v}_{j}^{(2 k+1)}\right\}_{j=1}^{|\hat{X}|}$ is a set of linear independent eigenvectors of $\mathbf{D}_{2 k+1}(\hat{X})$. Assume that $\lambda_{j}^{(2 k+1)}$ is an eigenvalue of $\mathbf{D}_{2 k+1}(\hat{X})$ with respect to the eigenvector $\mathbf{v}_{j}^{(2 k+1)}$. Since $\mathbf{D}_{2 k+1}(\hat{X})$ is a symmetric positive semidefinite matrix, we have $\lambda_{j}^{(2 k+1)} \geqslant 0$ for each $j \in\{1,2, \ldots,|\hat{X}|\}$. Then, according to (23), we have

$$
\left(1-|\hat{X}| \cdot f_{2 i+1}\right) \cdot \lambda \mathbf{v}=\lambda \cdot \sum_{k=\frac{t-2 i-1}{2}}^{\frac{s-2}{2}} f_{2 k+1} \cdot \mathbf{D}_{2 k+1}(\hat{X}) \sum_{j=1}^{|\hat{X}|} \mathbf{v}_{j}^{(2 k+1)},
$$

which implies

$$
\left(1-|\hat{X}| \cdot f_{2 i+1}\right) \cdot \lambda \mathbf{v}=\lambda \cdot \sum_{k=\frac{t-2 i-1}{2}}^{\frac{s-2}{2}} f_{2 k+1} \cdot \sum_{j: \lambda_{j}^{(2 k+1)}>0} \lambda_{j}^{(2 k+1)} \mathbf{v}_{j}^{(2 k+1)} .
$$

Thus, if $f_{2 i+1} \neq \frac{1}{|\hat{X}|}$, then $\mathbf{v}$ can be written as a linear combination of vectors in

$$
\sum_{k=\frac{t-2 i-1}{2}}^{\frac{s-2}{2}} V_{2 k+1}(\hat{X})
$$

which implies that $\mathbf{v} \in \sum_{k=\frac{t-2 i-1}{2}}^{\frac{s-2}{2}} V_{2 k+1}(\hat{X})$. Since $\mathbf{v}$ can be any vector in $V_{2 i+1}(\hat{X})$, we arrive at our conclusion. 
It remains to show that the coefficient $f_{t-s+2}$ in the Gegenbauer expansion of $F_{\hat{X}}(x)$ is not $\frac{1}{|\hat{X}|}$. We need the following lemma.

Lemma 16. [12, Lemma 2.6] Assume $F(x)=\sum_{k} f_{k} G_{k}^{(d)}(x)$ and let $Q(x):=\frac{G_{l}^{(d)}(x)}{h_{l}} \cdot F(x)$ for some positive integer $l$. Assume $Q(x)$ has the Gegenbauer expansion $Q(x)=\sum_{k} q_{k}$. $G_{k}^{(d)}(x)$. Then $q_{0}=f_{l}$.

With the help of the above lemma, we now show that the coefficient $f_{t-s+2}$ in the Gegenbauer expansion of $F_{\hat{X}}(x)$ is not $\frac{1}{|\hat{X}|}$. Actually, we prove that $f_{t-s+2}$ is the first coefficient with this property.

Lemma 17. Suppose $X \subset \mathbb{S}^{d-1}$ is an antipodal s-distance set with strength $t$, where $s \in\left[\frac{t+3}{2}, t+1\right]$ is an even integer and $t \geqslant 3$ is an odd integer. Let $\hat{X}$ be a half of $X$. Assume the annihilator polynomial of $\hat{X}$ has the Gegenbauer expansion $F_{\hat{X}}(x)=\sum_{k=0}^{\frac{s-2}{2}} f_{2 k+1} G_{2 k+1}^{(d)}(x)$. Then, $f_{t-s+2} \neq \frac{1}{|\hat{X}|}$ and $f_{l-s+2}=\frac{1}{|\hat{X}|}$ for each odd integer $l$ satisfying $s-1 \leqslant l<t$.

Proof. Set $Q_{l}(x):=\frac{G_{l-s+2}^{(d)}(x)}{h_{l-s+2}} \cdot F_{\hat{X}}(x)$ for each odd integer $l$ satisfying $s-1 \leqslant l \leqslant t$. Since $F_{\hat{X}}(x)$ is a polynomial of degree $s-1$, we see that $Q_{l}(x)$ is a polynomial of degree $l+1$. Noting that both $G_{l-s+2}^{(d)}(x)$ and $F_{\hat{X}}(x)$ are odd functions, we obtain that $Q_{l}(x)$ is an even function. Thus we can assume $Q_{l}(x)$ has the Gegenbauer expansion $Q_{l}(x)=$ $\sum_{i=0}^{\frac{l+1}{2}} q_{2 i}^{(l)} \cdot G_{2 i}^{(d)}(x)$. Since $F_{\hat{X}}(1)=1$ and $F_{\hat{X}}(\alpha)=0$ for each $\alpha \in A(\hat{X})$, we obtain that $Q_{l}(1)=1$ and $Q_{l}(\alpha)=0$ for each $\alpha \in A(\hat{X})$. This implies

$$
\sum_{\mathbf{x}, \mathbf{y} \in \hat{X}} Q_{l}(\langle\mathbf{x}, \mathbf{y}\rangle)=|\hat{X}|
$$

On the other hand, we have

$$
\sum_{\mathbf{x}, \mathbf{y} \in \hat{X}} Q_{l}(\langle\mathbf{x}, \mathbf{y}\rangle)=\sum_{\mathbf{x}, \mathbf{y} \in \hat{X}} \sum_{i=0}^{\frac{l+1}{2}} q_{2 i}^{(l)} \cdot G_{2 i}^{(d)}(\langle x, y\rangle)=|\hat{X}|^{2} q_{0}^{(l)}+\sum_{i=1}^{\frac{l+1}{2}}\left(q_{2 i}^{(l)} \cdot \sum_{\mathbf{x}, \mathbf{y} \in \hat{X}} G_{2 i}^{(d)}(\langle\mathbf{x}, \mathbf{y}\rangle)\right)
$$

Note that $\mathbf{H}_{0}(\hat{X})$ is the all-ones vector of size $|\hat{X}|$. According to (13), for each $i \in$ $\left\{1,2, \ldots, \frac{l+1}{2}\right\}$, we have

$$
\begin{aligned}
\sum_{\mathbf{x}, \mathbf{y} \in \hat{X}} G_{2 i}^{(d)}(\langle\mathbf{x}, \mathbf{y}\rangle) & =\mathbf{H}_{0}(\hat{X})^{T} \mathbf{D}_{2 i}(\hat{X}) \mathbf{H}_{0}(\hat{X}) \\
& =\mathbf{H}_{0}(\hat{X})^{T} \mathbf{H}_{2 i}(\hat{X}) \mathbf{H}_{2 i}(\hat{X})^{T} \mathbf{H}_{0}(\hat{X})=\left\|\mathbf{H}_{2 i}(\hat{X})^{\mathrm{T}} \mathbf{H}_{0}(\hat{X})\right\|_{2}^{2} .
\end{aligned}
$$

Combining (25) and (26), we obtain

$$
\sum_{\mathbf{x}, \mathbf{y} \in \hat{X}} Q_{l}(\langle\mathbf{x}, \mathbf{y}\rangle)=|\hat{X}|^{2} q_{0}^{(l)}+\sum_{i=1}^{\frac{l+1}{2}} q_{2 i}^{(l)} \cdot\left\|\mathbf{H}_{2 i}(\hat{X})^{\mathrm{T}} \mathbf{H}_{0}(\hat{X})\right\|_{2}^{2}
$$


Combining (24) and (27), we arrive at

$$
|\hat{X}|-|\hat{X}|^{2} q_{0}^{(l)}=\sum_{i=1}^{\frac{l+1}{2}} q_{2 i}^{(l)} \cdot\left\|\mathbf{H}_{2 i}(\hat{X})^{\mathrm{T}} \mathbf{H}_{0}(\hat{X})\right\|_{2}^{2} .
$$

Since $X$ has strength $t$, by Corollary 13 we have

$$
\left\|\mathbf{H}_{2 i}(\hat{X})^{\mathrm{T}} \mathbf{H}_{0}(\hat{X})\right\|_{2}^{2}=0, \text { for all } i \in\left\{1,2, \ldots, \frac{t-1}{2}\right\}
$$

and

$$
\left\|\mathbf{H}_{t+1}(\hat{X})^{\mathrm{T}} \mathbf{H}_{0}(\hat{X})\right\|_{2}^{2} \neq 0 .
$$

Then, by equation (28) we obtain

$$
|\hat{X}|-|\hat{X}|^{2} q_{0}^{(l)}=0
$$

when $s-1 \leqslant l<t$ and

$$
|\hat{X}|-|\hat{X}|^{2} q_{0}^{(t)}=q_{t+1}^{(t)} \cdot\left\|\mathbf{H}_{t+1}(\hat{X})^{\mathrm{T}} \mathbf{H}_{0}(\hat{X})\right\|_{2}^{2} .
$$

The (31) implies $q_{0}^{(l)}=\frac{1}{|\hat{X}|}$ for each odd integer $s-1 \leqslant l<t$. Noting that $Q_{t}(x)$ is a polynomial of degree $t+1$, we have $q_{t+1}^{(t)} \neq 0$. Combining (30) and (32), we obtain $q_{0}^{(t)} \neq \frac{1}{|\hat{X}|}$. By Lemma 16 we know that $q_{0}^{(l)}=f_{l-s+2}$ for each $l \geqslant s-1$. Hence, we arrive at our conclusion.

We next present a proof of Theorem 2 .

Proof of Theorem 2. Recall that $X$ is an antipodal $s$-distance set with strength $t$. Let $\hat{X}$ be a half of $X$. Combining Lemma 15 and Lemma 17, we know that $V_{t-s+2}(\hat{X})$ is contained in $V_{s-1}(\hat{X})$. Then by (18) we have

$$
\begin{aligned}
|\hat{X}| & \leqslant \operatorname{dim}\left(\sum_{k=0}^{\frac{s-2}{2}} V_{2 k+1}(\hat{X})\right)=\operatorname{dim}\left(\sum_{\substack{k=0 \\
k \neq \frac{t-s+1}{2}}}^{\frac{s-2}{2}} V_{2 k+1}(\hat{X})\right) \\
& \leqslant \sum_{\substack{k=0 \\
k \neq \frac{t-s+1}{2}}}^{\frac{s-2}{2}} \operatorname{dim} V_{2 k+1}(\hat{X}) \leqslant \sum_{k=0}^{\frac{s-2}{2}} h_{2 k+1}-h_{t-s+2}=\left(\begin{array}{c}
d+s-2 \\
s-1
\end{array}\right)-h_{t-s+2} .
\end{aligned}
$$

The last inequality in (33) follows from

$$
\operatorname{dim} V_{2 k+1}(X)=\operatorname{rank}\left(\mathbf{D}_{2 k+1}(X)\right) \leqslant \operatorname{rank}\left(\mathbf{H}_{2 k+1}(X)\right) \leqslant h_{2 k+1} .
$$

Noting that $|X|=2|\hat{X}|$, we obtain $|X| \leqslant 2\left(\begin{array}{c}d+s-2 \\ s-1\end{array}\right)-2 h_{t-s+2}$. 
Remark 18. Lemma 15 and Lemma 17 can be easily extended to the case when $s$ is an odd integer. Using these extended results one can obtain an upper bound on $|X|$ for odd $s \in\left[\frac{t+5}{2}, t+2\right]$, which is actually the same with the bound in (6). Hence, for clarity and convenience we only consider the case when $s$ is an even integer in Lemma 15 and Lemma 17.

Remark 19. Note that the framework of Nozaki and Suda developed in [23] can be applied to any finite two-point-homogeneous space. Since we follow the framework of Nozaki and Suda, our method in Theorem 2 can also be applied to antipodal designs with few angles in the binary Hamming schemes.

\section{Proof of Theorem 3}

The aim of this section is to present a proof of Theorem 3. We need the following necessary condition on the existence of real ETFs.

Lemma 20. (Theorem $\mathrm{A}$ in [25]) Let $d$ and $n$ be two integers satisfying $n>d+1>2$ and $n \neq 2 d$. If there exists an ETF for $\mathbb{R}^{d}$ with size $n$, then both $\sqrt{\frac{d(n-1)}{n-d}}$ and $\sqrt{\frac{(n-d)(n-1)}{d}}$ are odd integers.

Using the above lemma, we present a proof of Theorem 3.

Proof of Theorem 3. Recall that $\Phi$ is an ETF for $\mathbb{R}^{d}$ with size $n>d+1 \geqslant 6$. We first show that $n \leqslant \frac{d(d+2)}{3}$ if $n \neq \frac{d(d+1)}{2}$. Notice that $2 d<\frac{d(d+2)}{3}$ when $d \geqslant 5$, so we only need to consider the case when $n>2 d$. According to Lemma 20, we can assume $\sqrt{\frac{d(n-1)}{n-d}}=2 k-1$ for some positive integer $k>1$. Then a simple calculation shows:

$$
\begin{aligned}
(2 k-1)^{2}(n-d) & =d(n-1) \\
\left((2 k-1)^{2}-d\right)(n-d) & =d(d-1) .
\end{aligned}
$$

Since $n-d$ and $d(d-1)$ are positive, from $(34 \mathrm{~b})$ we obtain that $(2 k-1)^{2}-d$ is a positive integer. If $(2 k-1)^{2}-d=1$, then (34b) gives $n=d^{2}$. This is impossible since $n$ must satisfy the Gerzon bound (3). Hence, we must have $(2 k-1)^{2}-d \geqslant 2$. If $(2 k-1)^{2}-d=2$, then $(34 \mathrm{~b})$ gives $n=\frac{d(d+1)}{2}$; otherwise, we have $(2 k-1)^{2}-d \geqslant 3$, then (34b) implies $n \leqslant \frac{d(d+2)}{3}$. Hence, we have $n \leqslant \frac{d(d+2)}{3}$ if $n \neq \frac{d(d+1)}{2}$.

It remains to prove that $n \geqslant d+\frac{1}{2}+\sqrt{3 d+\frac{1}{4}}$ if $n \neq d+\frac{1}{2}+\sqrt{2 d+\frac{1}{4}}$. Notice that $d+\frac{1}{2}+\sqrt{3 d+\frac{1}{4}}<2 d$ when $d \geqslant 5$, so we only need to consider the case when $n<2 d$. Set $m:=n-d$. By Lemma 20, we may assume $\sqrt{\frac{m(n-1)}{n-m}}=\sqrt{\frac{(n-d)(n-1)}{d}}=2 p-1$ for some positive integer $p$. By similar computation with (34b) we obtain

$$
\left((2 p-1)^{2}-m\right)(n-m)=m(m-1) .
$$


Since $n-m=d>0$ and $m=n-d>1$, from (35) we see that $(2 p-1)^{2}-m$ is a positive integer. If $(2 p-1)^{2}-m=1$, then (35) gives $n=m^{2}$, that is, $n=d+\frac{1}{2}+\sqrt{d+\frac{1}{4}}$. This is impossible since $n$ must satisfy the Gerzon bound (3). Hence, we have $(2 p-1)^{2}-m \geqslant 2$. If $(2 p-1)^{2}-m=2$, then we have $n=\frac{m(m+1)}{2}$, that is, $n=d+\frac{1}{2}+\sqrt{2 d+\frac{1}{4}}$; otherwise, we have $(2 p-1)^{2}-m \geqslant 3$, then (35) implies $n \leqslant \frac{m(m+2)}{3}$, that is, $n \geqslant d+\frac{1}{2}+\sqrt{3 d+\frac{1}{4}}$. Hence, we must have $n \geqslant d+\frac{1}{2}+\sqrt{3 d+\frac{1}{4}}$ if $n \neq d+\frac{1}{2}+\sqrt{2 d+\frac{1}{4}}$. Putting all these together, we arrive at the conlcusion.

For the remainder of this section we compare the Krein conditions for strongly regular graph with Gerzon bound. It is well known that there exists an ETF for $\mathbb{R}^{d}$ with size $n>d+1>2$ if and only if there exists a strongly regular graph with parameters $\left(n-1, a, \frac{3 a-n}{2}, \frac{a}{2}\right)[25,29,14]$, where $a$ is defined in (9). It is also known that each strongly regular graph satisfies the following Krein conditions:

Lemma 21. (see $[24,8,14]$ ) Assume there exists a strongly regular graph $\Gamma$ with given parameters $v, k, \lambda, \mu$. Then the parameters $v, k, \lambda, \mu$ satisfy the following Krein conditions:

$$
\begin{aligned}
& K_{1}:=\left(k+r_{1}\right)\left(r_{2}+1\right)^{2}-\left(r_{1}+1\right)\left(k+r_{1}+2 r_{1} r_{2}\right) \geqslant 0, \\
& K_{2}:=\left(k+r_{2}\right)\left(r_{1}+1\right)^{2}-\left(r_{2}+1\right)\left(k+r_{2}+2 r_{1} r_{2}\right) \geqslant 0,
\end{aligned}
$$

where $r_{1}$ and $r_{2}$ are defined in (15).

Hence, if we apply the above lemma to strongly regular graphs with parameters $(n-$ $\left.1, a, \frac{3 a-n}{2}, \frac{a}{2}\right)$, then (36a) and (36b) provide two necessary conditions on the existence of nontrivial ETFs. The authors of [29] and [14] wondered whether these two necessary conditions are covered by the Gerzon bound (3) or other known necessary conditions. In what follows we show that they are actually equivalent to the Gerzon bound (3).

Proposition 22. Assume that $n>d+1>2$. Set

$$
a:=\frac{n}{2}-1+\left(1-\frac{n}{2 d}\right) \sqrt{\frac{d(n-1)}{n-d}} .
$$

The $(n, d)$ satisfies Krein conditions (36a) and (36b) with parameters $v=n-1, k=$ $a, \lambda=\frac{3 a-n}{2}, \mu=\frac{a}{2}$ if and only if $(n, d)$ satisfies the Gerzon bound (3).

Proof. Substituting $v=n-1, k=a, \lambda=\frac{3 a-n}{2}, \mu=\frac{a}{2}$ into equation (15), we can represent $r_{1}$ and $r_{2}$ as follows:

$$
\begin{aligned}
& r_{1}=\frac{1}{2} \cdot \sqrt{\frac{d(n-1)}{n-d}}-\frac{1}{2} \\
& r_{2}=-\frac{1}{2} \cdot \frac{n-d}{d} \cdot \sqrt{\frac{d(n-1)}{n-d}}-\frac{1}{2} .
\end{aligned}
$$


Next, substituting equation (37), (38a), (38b) into (36a) and (36b), we obtain

$$
\begin{aligned}
& K_{1}=\frac{n}{8 d} \cdot \sqrt{\frac{n-1}{n-d}} \cdot\left(\sqrt{\frac{n-1}{n-d}}-\sqrt{\frac{1}{d}}\right) \cdot\left(n^{2}-(2 d+1) n+d^{2}-d\right), \\
& K_{2}=\frac{n}{8 d(n-d)} \cdot\left(n-1+\sqrt{\frac{d(n-1)}{n-d}}\right) \cdot\left(d^{2}+d-2 n\right) .
\end{aligned}
$$

Since $n>d+1>2, K_{1} \geqslant 0$ if and only if $n^{2}-(2 d+1) n+d^{2}-d \geqslant 0$, i.e., $n \geqslant d+\frac{1}{2}+$ $\sqrt{2 d+\frac{1}{4}}$. Moreover, $K_{2} \geqslant 0$ if and only if $n \leqslant \frac{d(d+1)}{2}$. We arrive at the conclusion.

\section{$5 \quad$ Proof of Theorem 7}

The main goal of this section is to prove Theorem 7. For convenience, in the rest of this paper, we use $\alpha_{n, d}$ to denote the Levenstein bound in (2b), i.e.,

$$
\alpha_{n, d}:=\sqrt{\frac{3 n-d(d+2)}{(d+2)(n-d)}} .
$$

We assume that $\Phi \subset \mathbb{S}^{d-1}$ with $|\Phi|=n$ is a Levenstein-equality packing, i.e., $\mu(\Phi)=\alpha_{n, d}$. Hence $\Phi$ has the angle set $\left\{0, \alpha_{n, d},-\alpha_{n, d}\right\}$ (see [12, Example 8.4] and [15, Proposition 3.3]).

We begin with introducing two basic properties about Levenstein-equality packings. The following lemma says that each Levenstein-equality packing gives rise to a strongly regular graph.

Lemma 23. [22, Page 83] Assume $\Phi=\left\{\boldsymbol{\varphi}_{i}\right\}_{i=1}^{n} \subset \mathbb{S}^{d-1}$ is a Levenstein-equality packing with the angle set $\left\{0, \alpha_{n, d},-\alpha_{n, d}\right\}$, where $\alpha_{n, d}$ is defined in (40). Let $\Gamma$ be a graph with $n$ vertices where vertex $i$ and vertex $j$ are adjacency if $\left\langle\boldsymbol{\varphi}_{i}, \boldsymbol{\varphi}_{j}\right\rangle \neq 0$. Then $\Gamma$ is a strongly regular graph with parameters $(n, k, \lambda, \mu)$, where

$$
k=-\frac{r_{2}\left(3 \cdot r_{1}-r_{2}\right)}{2}, \lambda=r_{1}+r_{2}+\mu, \mu=-\frac{r_{2}\left(r_{1}-r_{2}\right)}{2}, r_{1}=\frac{2 n}{3 d}+\frac{r_{2}}{3}-\frac{2}{3}, r_{2}=-\frac{1}{\alpha_{n, d}^{2}} .
$$

Here, $r_{1}$ and $r_{2}$ are defined in (15).

Remark 24. Substituting (40) into (41) we can write these parameters in terms of $n$ and $d$ as follows:

$$
\begin{aligned}
k & =\frac{(n-d)^{2}(d+2)}{d \cdot(3 n-d(d+2))} \\
\lambda & =\frac{(n-d) \cdot\left((d+8) n^{2}-9 d(d+2) n+2 d^{2}(d+2)^{2}\right)}{d \cdot(3 n-d(d+2))^{2}} \\
\mu & =\frac{(n-d)^{2}(d+2) n}{d \cdot(3 n-d(d+2))^{2}} \\
r_{1} & =\frac{(n-d)(2 n-d(d+2))}{d \cdot(3 n-d(d+2))}, r_{2}=-\frac{(n-d)(d+2)}{3 n-d(d+2)}
\end{aligned}
$$


Recall that $r_{1}$ and $r_{2}$ are the eigenvalues of $\mathbf{A}$, which is the adjacency matrix of $\Gamma$, with multiplicities $n_{1}$ and $n_{2}$. Substituting (42a), (42b) and (42c) into (16), we can obtain the multiplicities of $r_{1}$ and $r_{2}$ as follows

$$
n_{1}=\frac{d(d+1)}{2}-1, n_{2}=n-\frac{d(d+1)}{2} .
$$

The next lemma introduces another property of Levenstein-equality packings.

Lemma 25. Let $d \geqslant 4$ be an integer. If $\Phi=\left\{\boldsymbol{\varphi}_{i}\right\}_{i=1}^{n} \subset \mathbb{S}^{d-1}$ is a Levenstein-equality packing with size $n$, then both $\frac{1}{\alpha_{n, d}}$ and $\frac{n-d}{d \cdot \alpha_{n, d}}$ are integers.

Proof. Let M be the matrix of size $d \times n$ whose $i$-th column is $\boldsymbol{\varphi}_{i}$. Since $\Phi$ is a Levensteinequality packing, $\Phi \cup-\Phi$ is an antipodal spherical 5-design. Note that every spherical 2-design is a unit norm tight frame [30, Proposition 6.1]. Hence, $\Phi$ forms a unit norm tight frame in $\mathbb{R}^{d}$, i.e., $\mathbf{M M}^{\mathrm{T}}=\frac{n}{d} \cdot \mathbf{I}_{d \times d}$ which has eigenvalue $n / d$ with multiplicities $d$. Set $\mathbf{G}:=\frac{1}{\alpha_{n, d}} \cdot\left(\mathbf{M}^{\mathrm{T}} \mathbf{M}-\mathbf{I}_{n \times n}\right)$. Since the nonzero eigenvalues of $\mathbf{M}^{\mathrm{T}} \mathbf{M}$ and $\mathbf{M} \mathbf{M}^{\mathrm{T}}$ have the same value and the same algebraic multiplicity, we see that $\mathbf{G}$ has two different eigenvalues:

$$
\lambda_{1}=-\frac{1}{\alpha_{n, d}} \quad \text { and } \quad \lambda_{2}=\frac{1}{\alpha_{n, d}} \cdot \frac{n-d}{d}
$$

with multiplicities $n-d$ and $d$, respectively. Moreover, since the $(i, j)$-entry of $\mathbf{M}^{\mathrm{T}} \mathbf{M}$ is the inner product between $\boldsymbol{\varphi}_{i}$ and $\boldsymbol{\varphi}_{j}, \mathbf{G}$ is a matrix whose diagonal entries are all zeros and non-diagonal entries are 0 or \pm 1 . This means that both $\lambda_{1}$ and $\lambda_{2}$ are algebraic integers. Since an algebraic integer is an integer if it is a rational number, it remains to prove that both $\lambda_{1}$ and $\lambda_{2}$ are rational numbers. For the aim of contradiction, we assume that $\lambda_{1}$ is irrational. Let $f(x)$ be the minimal polynomial of $\lambda_{1}$. Then the characteristic polynomial of $\mathbf{G}$ is divided by $f(x)^{n-d}$. This means that any algebraic conjugate of $\lambda_{1}$ is also an eigenvalue of $\mathbf{G}$ with multiplicity $n-d$. However, since $\Phi$ is a Levenstein-equality packing, we have $n>\frac{d(d+1)}{2}$. Combining with $d \geqslant 4$, we have $d<n-d$, meaning that $\mathbf{G}$ does not have two eigenvalues with the same multiplicity. This is a contradiction. Hence, $\lambda_{1}$ is rational. Since $\lambda_{2}=-\lambda_{1} \cdot \frac{n-d}{d}$, we obtain that $\lambda_{2}$ is also rational. This completes the proof.

Remark 26. From viewpoint of the association scheme, the authors in [2, Theorem 8.1] showed that $\frac{1}{\alpha_{n, d}}$ is an integer if the strongly regular graph generated by $\Phi$ in Lemma 23 is not a conference graph. Recall that a strongly regular graph is a conference graph if and only if its parameters are $\left(n, \frac{n-1}{2}, \frac{n-5}{4}, \frac{n-1}{4}\right)$. A simple calculation shows that the strongly regular graph with parameters described in (42) can never be a conference graph. Hence, the results in $[2$, Theorem 8.1$]$ also imply the integrality of $\frac{1}{\alpha_{n, d}}$.

Now we are ready to prove Theorem 7 .

Proof of Theorem \%. Recall that $\Phi \subset \mathbb{S}^{d-1}$ has the angle set $A(\Phi)=\left\{0, \alpha_{n, d},-\alpha_{n, d}\right\}$, where $\alpha_{n, d}$ is defined in (40). We claim that $n \geqslant \frac{d(d+3)}{2}$ if $n \neq \frac{d(d+2)}{2}$. According to Lemma 
25, we can assume $\alpha_{n, d}=\frac{1}{k}$ for some positive integer $k$. Noting $\alpha_{n, d}=\sqrt{\frac{3 n-d(d+2)}{(d+2)(n-d)}}=\frac{1}{k}$, we have

$$
3 k^{2} \cdot\left(n-\frac{d(d+2)}{3}\right)=(d+2)(n-d)
$$

which implies

$$
\left(3 k^{2}-d-2\right)\left(n-\frac{d(d+2)}{3}\right)=\frac{1}{3} \cdot d(d-1)(d+2) .
$$

We set $\alpha:=3 k^{2}-d-2 \in \mathbb{Z}$. Since either $n \geqslant \frac{d(d+3)}{2}$ or $n=\frac{d(d+2)}{2}$, we have $n>\frac{d(d+2)}{3}$. Then (45) implies that $\alpha \in \mathbb{Z}_{+}$and

$$
n=\frac{d(d+2)(d-1+\alpha)}{3 \alpha} .
$$

We next show that $\alpha \in\left[2, \frac{2(d-1)(d+2)}{d+5}\right] \cap \mathbb{Z}$ if $n \neq \frac{d(d+2)}{2}$. Indeed, $\alpha \leqslant \frac{2(d-1)(d+2)}{d+5}$ follows from $n \geqslant \frac{d(d+3)}{2}$. We still need show that $\alpha \geqslant 2$. For the aim of contradiction, we assume that $\alpha=1$. Then (46) implies $n=\frac{d^{2}(d+2)}{3}$. Noting that $\Phi \cup-\Phi$ is an antipodal 4-distance set, we have $|\Phi \cup-\Phi|=2 n=\frac{2 d^{2}(d+2)}{3}$. On the other hand, according to Delsarte-GoethalsSeidel bound, i.e. (1), we have $|\Phi \cup-\Phi| \leqslant \frac{d(d+1)(d+2)}{3}$. We have $\frac{2 d^{2}(d+2)}{3} \leqslant \frac{d(d+1)(d+2)}{3}$, which is a contradiction.

We still need show $n \geqslant \frac{d(d+3)}{2}$ if $n \neq \frac{d(d+2)}{2}$. We assume $n \neq \frac{d(d+2)}{2}$. According to Lemma $23, \Phi$ gives a strongly regular graph $\Gamma$ with parameters described in (42). Let $E_{2}$ denote the eigenspace of the adjacency matrix of $\Gamma$ with respect to the eigenvalue $r_{2}$, and let $Y$ denote the spherical embedding of $\Gamma$ with respect to $E_{2}$. Since $Y$ is obtained by orthogonally projecting a standard basis of $\mathbb{R}^{n}$ onto the eigenspace $E_{2}$ and rescaling to have unit norm, we know that $Y \subset \mathbb{S}^{n_{2}-1}$. Combining with (43), we have $Y \subset \mathbb{S}^{n-\frac{d(d+1)}{2}}-1$. By substituting (42) into (17), we see that $Y$ is a spherical two-distance set with the angle set $\left\{-\frac{d}{n-d}, \frac{d}{2 n-d(d+1)}\right\}$. Since the Levenstein bound (2b) is attained only if $n>\frac{d(d+1)}{2}[20$, Theorem 6.13], we have $-\frac{d}{n-d}<0$. Also note that $\frac{d}{2 n-d(d+1)} \neq 1$ since $n \neq \frac{d(d+2)}{2}$. Hence, $Y$ contains no repeated vectors. According to the Delsarte-Goethals-Seidel bound for spherical two-distance sets (see [12, Theorem 4.8]), we have

$$
|Y|=n \leqslant \frac{\left(n-\frac{d(d+1)}{2}\right)\left(n-\frac{d(d+1)}{2}+3\right)}{2} .
$$

Rearranging the terms in (47) and solving a quadratic inequality gives $n \geqslant \frac{d(d+3)}{2}$ or $n \leqslant \frac{(d-2)(d+1)}{2}$. Since $n>\frac{d(d+1)}{2}$, we obtain $n \geqslant \frac{d(d+3)}{2}$. Hence, we have $n \geqslant \frac{d(d+3)}{2}$ if $n \neq \frac{d(d+2)}{2}$.

\section{References}

[1] Ei. Bannai and Et. Bannai. A survey on spherical designs and algebraic combinatorics on spheres. European J. Combin., 30 (6) (2009), 1392-1425. 
[2] Ei. Bannai and Et. Bannai. Spherical designs and Euclidean designs. In Recent Developments in Algebra and Related Areas, Beijing, 2007, Adv. Lect. Math., 8, Higher Education Press, Beijing; International Press, Boston, (2009), 1-37.

[3] Ei. Bannai and R. Damerell. Tight spherical designs I. J. Math. Soc. Japan., 31 (1979), 199-207.

[4] Ei. Bannai, A. Munemasa, and B. Venkov. The nonexistence of certain tight spherical designs. European J. Combin., Algebra i Analiz 16:4 (2004), 1-23.

[5] Ei. Bannai, Et. Bannai, Z. Xiang, W. Yu, and Y. Zhu. Classification of Spherical 2-distance $\{4,2,1\}$-designs by Solving Diophantine Equations. Taiwanese J. Math., 25:1 (2021) 1-22 https://doi.org/10.11650/tjm/200601.

[6] D. Bilyk, A. Glazyrin, R. Matzke, J. Park, and O. Vlasiuk. Optimal measures for p-frame energies on spheres. arXiv:1908.00885. (2019)

[7] A.V. Bondarenko, A. Prymak, and D. Radchenko. Non-existence of $(76,30,8,14)$ strongly regular graph. Linear Algebra Appl., 527 (2017), 53-72

[8] A. E. Brouwer and W. H. Haemers. Spectra of graphs. Springer Science \& Business Media, 2011.

[9] P.J. Cameron. Strongly regular graphs. Topics in Algebraic Graph Theory, 102 (2004), 203-221.

[10] J. H. Conway, R. H. Hardin, and N. J. A. Sloane. Packing lines, planes, etc.: packings in Grassmannian spaces. Experiment. Math., 5(2)(1996), 139-159.

[11] H. Cohn and A. Kumar. Universally optimal distribution of points on spheres. J. Amer. Math. Soc., 20 (2007), 99-148.

[12] P. Delsarte, J. M. Goethals, and J. J. Seidel. Spherical codes and designs. Geometriae Dedicata, 6 (1977), .363-388.

[13] M. Fickus, J. Jasper, and D. G. Mixon. Packings in real projective spaces. SIAM J. Appl. Algebra Geometry., 2(3), (2018), 377-409.

[14] M. Fickus, and D. G. Mixon. Tables of the existence of equiangular tight frames. arXiv: $1504.00253(2015)$

[15] J. I. Haas, N. Hammen, and D. G. Mixon. The Levenstein bound for packings in projective spaces. Wavelets and Sparsity XVII, Vol. 10394, p. 103940V (24 August 2017), International Society for Optics and Photonics. https://doi.org/10.1117/12.2275373

[16] R. H. Hardin, and N. J. A. Sloane. McLaren's improved snub cube and other new spherical designs in three dimensions. Discrete Comput. Geom., 15 (1996), 429-441. https://doi.org/10.1007/BF02711518

[17] J. Jasper, E. J. King, and D. G. Mixon. Game of Sloanes: best known packings in complex projective space. Wavelets and Sparsity XVIII, vol. 11138, p. 111381E. International Society for Optics and Photonics, 2019.

[18] P. W. H. Lemmens and J. J. Seidel. Equiangular lines. J. Algebra, 24 (1973) 494-512. 
[19] V. I. Levenshtein. Designs as maximum codes in polynomial metric spaces. Acta Appl. Math., 29 (1992), 1-82.

[20] V. I. Levenshtein. Universal bounds for codes and designs. Handbook of coding theory, 1 (1998), 499-648.

[21] A. Munemasa. Spherical Designs, in: Handbook of Combinatorial Designs, 2nd ed., C. J. Colbourn, J. H. Dinitz, eds. CRC Press, (2007), 617-622.

[22] A. Neumaier. Combinatorial configurations in terms of distances. Memorandum 81-09 (Dept. of Mathematics), (1981), Eindhoven University of Technology.

[23] H. Nozaki and S. Suda. Bounds on s-distance sets with strength t. SIAM J. Discrete Math., 25(4), (2011), 1699-1713.

[24] L. L. Scott Jr. A condition on Higman's parameters. Notices of the American Mathematical Society, 20 (1973), A-97.

[25] M. A. Sustik, J. A. Tropp, I. S. Dhillon, and R. W. Heath. On the existence of equiangular tight frames. Linear Algebra Appl., 426, (2007), 619-635.

[26] G. Szegö. Orthogonal polynomials (Vol. 23). American Mathematical Soc., (1939).

[27] D. E. Taylor. Regular 2-graphs. Proc. London Math. Soc., (3), 35(2),(1977), 257-274.

[28] J. C. Tremain. Concrete Constructions of Real Equiangular Line Sets. arXiv:0811.2779 (2008)

[29] S. Waldron. On the construction of equiangular frames from graphs. Linear Algebra Appl., 431, (2009), 2228-2242.

[30] S. Waldron. An introduction to finite tight frames. Birkhäuser/Springer, New York, 2016.

[31] L. Welch. Lower bounds on the maximum cross correlation of signals. IEEE Trans. Inform. Theory., 20(3),(1974), 397-399. 